\title{
Discriminative Power of Lymphoid Cell Features: Factor Analysis Approach'
}

\author{
Igor Gurevich ${ }^{1}$, Dmitry Harazishvili ${ }^{2}$, Irina Jernova ${ }^{1}$, \\ Alexey Nefyodov ${ }^{1}$, Anastasia Trykova ${ }^{1}$, and Ivan Vorobjev ${ }^{2}$ \\ ${ }^{1}$ Scientific Council "Cybernetics" of the Russian Academy of Sciences \\ 40, Vavilov str., 119991 Moscow, GSP-1, Russian Federation \\ igourevi@ccas.ru \\ 2 Hematological Scientific Center of the Russian Academy of Medical Sciences \\ 4a, Novyi Zykovskii pr., 125167 Moscow, Russian Federation \\ ivorobjev@mail.ru
}

\begin{abstract}
The new results of the research in the field of automation of hematopoietic tumor diagnostics by analysis of the images of cytological specimens are presented. Factor analysis of numerical diagnostically important features used for the description of lymphoma cell nucleus was carried out in order to evaluate the significance of the features and to reduce the considered feature space. The following results were obtained: a) the proposed features were classified; b) the feature set composed of 47 elements was reduced to 8 informative factors; c) the extracted factors allowed to distinguish some groups of patients. This implies that received factors have substantial medical meaning. The results presented in the paper confirm the advisability of involving factor analysis in the automated system for morphological analysis of the cytological specimens in order to create a complex model of phenomenon investigated.
\end{abstract}

\section{Introduction}

In this paper, we describe new results of the research into automation of hematopoietic tumors diagnostics on the base of analysis of the images of cytological specimens. This work has been conducted since 2000 by the researchers of the Scientific Council "Cybernetics" of the Russian Academy of Sciences together with the researchers of the Hematological Scientific Center of the Russian Academy of Medical Sciences [1]. The necessary condition of such automation is the development of information technology for morphological analysis of the lymphoid cell nuclei of patients with hematopoietic tumors, which could be implemented in corresponding software system for automated diagnostics. The paper is devoted to the investigation of the numerical features used for the description of lymphoma cell nucleus by means of factor analysis. The method of factor analysis, which allows reducing and structur-

1 This work was partly supported by the Russian Foundation for Basic Research (grant No. 01-0790016, and 03-07-90406) and by Federal Target-Oriented Program "Research and Development in the Priority Directions of Science and Technology" in 2002-2006 (project No. 37.0011.11.0016).

A. Sanfeliu and J. Ruiz-Shulcloper (Eds.): CIARP 2003, LNCS 2905, pp. 298-305, 2003.

(C) Springer-Verlag Berlin Heidelberg 2003 
ing the initial data, proved to be efficient for the problem of morphological analysis of lymphocyte nucleus.

The paper is organized as follows. Section 2 contains a brief description of the developed information technology for the morphological analysis of the cytological specimens. Section 3 contains information about the initial data for factor analysis and about methods used for factor analysis. The results of factor analysis and some conclusions are presented in section 4 . Note that the developed technology is described entirely in [4].

\section{The Main Stages of the Morphological Analysis of the Blood Cells}

The developed information technology for the morphological analysis of the cytological specimens includes the following stages of data preparation and analysis:

1. Creation of a database containing images of specimens of lymphatic tissues with isolated lymphocyte nuclei for patients with different lymphoid tumors.

2. Normalization of the images in order to compensate for different illumination conditions and different colors of stain used for the specimens.

3. Choice of features which capture morphological characteristics of lymphocytes nuclei useful for lymphoma diagnostics.

4. Calculation of values, statistical and qualitative analysis of the chosen features for the set of available nuclei.

5. Selection of features for generating feature descriptions of lymphoma cell nuclei.

6. Cluster analysis of the nuclei by using different subsets of the generated set of features.

7. Qualitative and quantative analysis of the obtained clusters.

8. Formation of a new feature space for description of the patients:

- the 'large' clusters of the cell nuclei are selected;

- the new features are relational numbers of patient's nuclei that belong to the selected clusters.

9. Diagnosing the patients by the use of efficient recognition algorithms (for example, recognition algorithms based on estimate calculation [5]) applied to the feature descriptions developed in p.9.

\subsection{General Characteristic of the Source Data}

A base of photomicrographic images of lymphatic tissue imprints was created to select and describe diagnostically important features of lymphocyte nuclei images. The base contains 1585 photos of specimens of 36 patients. We choose 25 cases of aggressive lymphoid tumors (de novo large and mixed cell lymphomas (L) and transformed chronic lymphocytic leukemia (TCLL)) and 10 cases of indolent chronic lymphocytic leukemia (CLL). In one case the reactive lymphoid hyperplasia was diagnosed. 


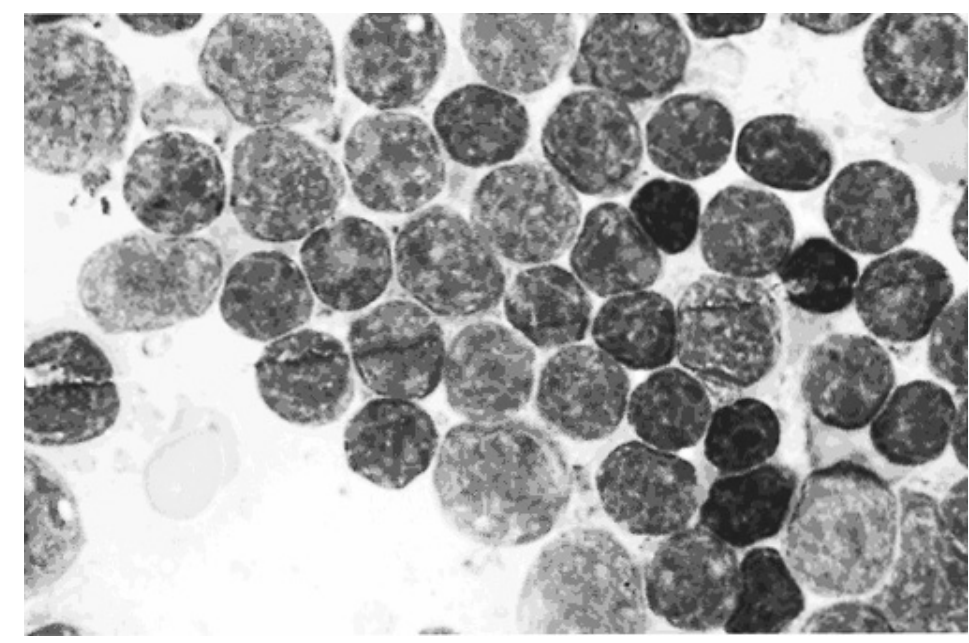

Fig. 1. Monochrome photomicrographic image of the footprint of lymphatic gland. Objective $\times 100$.

The photos of specimens were collected and stored as the RGB-images in 24-bit TIFF format. Figure 1 shows monochrome picture of the slide of lymphatic gland. On the original RGB-images 4327 nuclei of lymphocytic cells important for diagnostics were indicated by experts. These nuclei were further segmented and analyzed.

\subsection{Selection and Extraction of Features for Lymphocyte Nuclei Description}

The principal property of the proposed technology is the generation of lymphocyte nuclei description by the features chosen and calculated from the images of specimens by the methods of image processing and analysis, and also by the methods of mathematical morphology and Fourier analysis.

During the morphological analysis of lymphocyte nuclei, hematologists use the following characteristics.

1. Nuclei size and density of different lymphoid cells in the specimen (presence of cells with nucleus larger than in most cells).

2. Nuclear form (round, oval, folded), presence of invaginations.

3. Textural features of chromatin (dispersed or condensed pattern; if dispersed visual diameter of chromatin fibrils).

4. Presence or absence of nucleoli; if present - their number, size, form and location in nuclei (central or peripheral).

We provided formal equivalents of some of the above characteristics. They can be translated into geometrical and textural features of cell nuclei. Thus, the following 47 features were chosen to describe nuclei morphology:

1) an area of nucleus in pixels;

2) four statistical features calculated on nucleus brightness histogram (average, dispersion, $3^{\text {rd }}$ and $4^{\text {th }}$ central moments); 
3) 16 granulometric features of nucleus;

4) 26 features calculated on the Fourier spectrum of nucleus.

\subsection{Cluster Analysis of the Nuclei}

To preprocess the images of the specimens and to calculate the nuclei' features, a program was developed which uses the libraries of the "Black Square" software system [3]. After calculation of the features, their statistical and qualitative analysis was carried out. Conducted analysis allowed us to select several groups of features, which further yielded diagnostically-perspective taxonomies.

The main results of the lymphocytic cells investigations are based on their cluster analysis using developed feature space and its subspaces with the help of FOREL algorithm [2].

A number of sets of clusters were received by using different sets of features and different values of parameter of FOREL algorithm. Obtained sets of clusters were evaluated using various criteria (e.g., the number and the size of clusters in each set, the character of nuclei distribution in large clusters, etc.). Several "interesting" sets of clusters were selected which have good formal characteristics of their clusters and are promising for interpretation by hematologists.

The obtained results showed that:

1) the set of diagnostically important nuclei of the patients with considered lymphoid tumors is substantially heterogeneous since different clusters in it were clearly identified;

2) clusterization of the lymphocytic nuclei using developed feature set is important from the medical standpoint;

3) formal nucleus characterization by the developed feature set corresponds well with its qualitative morphological description and serves as a basis for development the automated software systems of morphological analysis of the blood cells and diagnosis of hemoblastoses;

4) the suggested technology provides transition from the diagnostic analysis of lymphocytes nuclei to diagnosing patients with hematopoietic tumors by the use of pattern recognition techniques.

\section{Factor Analysis of the Features of Lymphocyte Nuclei}

As it is known, factor analysis allows one to estimate the dimensionality of a set of observed variables and to determine the structure of interconnections between these variables. Factor analysis can be used to replace a large set of observed variables with a smaller set of new hypothetical variables called factors. These factors are treated as principal variables that truly describe investigated phenomenon.

The initial data were represented as a table with the rows corresponding to the nuclei' feature descriptions. Factor analysis was conducted on the whole of given nucleus, on different combinations of nucleus taken from patients with different forms 
of hemoblastoses (L, CLL and TCLL), and on 5 large clusters, extracted in the set of nucleus by means of taxonomy method.

The tables of nucleus feature descriptions were normalized by columns by the rule

$$
z_{i j}=\frac{y_{i j}-\overline{y_{j}}}{s_{j}},
$$

where $z_{i j}, y_{i j}$ are the normalized and primary values of the $j$ th feature of the $i$ th nucleus, $s_{j}$ is a standard deviation of the $j$ th feature, $\bar{y}_{j}$ is an average value of the $j$ th feature. As a result of such transformation the features' variances became unit. Then, the correlations of the features were calculated in each group of nuclei. Factor analysis was applied to the corresponding reduced correlation matrices with the communities on the principal diagonal. In some cases iterative procedure was applied for evaluation of communities, or squared coefficient of multiple correlation of features with the other ones was considered. The extraction of the factors was realized by means of 3 methods, namely by means of principal-factor method, centroid method and maximum-likelihood method. The quantity of the factors was determined as a result of combining the Kaizer criterion and the scree-test. The varimax-rotate strategy was applied with the purpose of obtaining the contansive interpretation of factors.

It is known that factor problem has an ambiguous solution depending on restrictions imposed on (the choice of factorial method). But considering that a single simple factor explanation lay in the very data, different methods should yield nearly the same mappings, and it signifies that it is possible to establish a certain correspondence between factors from different solutions. Thus, next stage of analysis was the search of correspondences between factor mappings received with the help of 3 different methods. Then average factor loadings of each feature under 3 methods were calculated, and factors were ranked by a maximal percent of variance explained by them.

The conducted factor analysis allowed classifying different numerical features according to the cross correlation in independent groups, defining dominant factors. As a matter of fact each new factor proved to be a linear combination of several initial features, signed with high loadings (exceeding 0,7) for this factor (the most informative features). The additional analysis revealed that a choice of the value 0,7 as a threshold was advisable as long as the combination of features with high loadings did not vary significantly under decrease of this value.

\section{The Results of Factor Analysis}

The results of factor analysis are presented in Table 1. The factor mappings received on the different groups of cell nucleus are presented in the columns of Table 1, and the factors of the same significance level are presented in the rows. The increase of row number corresponds to a reduction of statistical significance of the factor, which is determined in turn by decrease of the variance of this factor. Thus, we come to the following conclusions:

1. The initial set of features (47 elements) breaks at the average into 8 significant groups - factors. There are some cases where 3 factors of the greatest information 
density (which explain the largest part of the total variance of the features) combine into a single factor (general distribution of features by factors) in the group of patients with TCLL diagnosis and in the first cluster; and into 2 factors as well - in the groups of patients with CLL and L diagnosis. Due to mentioned above the quantity of important factors varies. The increase of the quantity of factors on the samples of nucleus of the smaller size may be explained by the following rule: if the dimensionality of the considered sample decreases until a certain moment the accuracy of its description with the help of the greater number of factors increases.

2. The factor mappings explain at the average $75 \%$ of the total variance of the features.

3. The analysis conducted allows establishing the following classification of the features.

a. The main factors include nearly all texture features, the feature with the number 1 (an area of nucleus in pixels) and 3 granulometric features with the numbers 14, 15, 16 (the general number of light grains in nuclei, the number of grains with typical size and with minimal size respectively). The features included in the main factors are of the greatest importance for hematopoietic tumors diagnostics and these very features should be considered in the first place.

b. The second dominant factor includes the statistical features with the numbers $3,4,5$ - variance, $3^{\text {rd }}$ and $4^{\text {th }}$ central moments calculated on nucleus brightness histogram respectively.

c. The features with the numbers 6, 7 (average and variance calculated on nucleus size histogram respectively) fall into the same factor with a minor statistical significance as a rule.

d. Granulometric features with the numbers 10, 18 (the number of grains with sizes corresponding to local maxima and local minima of the constructed functions) occur together as well.

The coefficients of cross correlation of the features are high enough in each factor.

4. It appeared that there are features that are inessential (have minor factor loadings) in all kinds of analysis, so these features bring in a little of new information. All these variables belong to the class of granulometric features of the nuclei and have the numbers $8,9-3^{\text {rd }}$ and $4^{\text {th }}$ central moments calculated on nucleus brightness histogram, 11, 12, 13 - typical, minimal and maximal size of light grains in the nuclei respectively. The features with the numbers $9,11,12$, and 13 have minor pair correlations with the other parameters stably $(<0,7)$.

5. The clusters and groups of patients are distinguished by the factors. It is important to note that some groups of nucleus contain features which don't occur in the set of the other groups. 


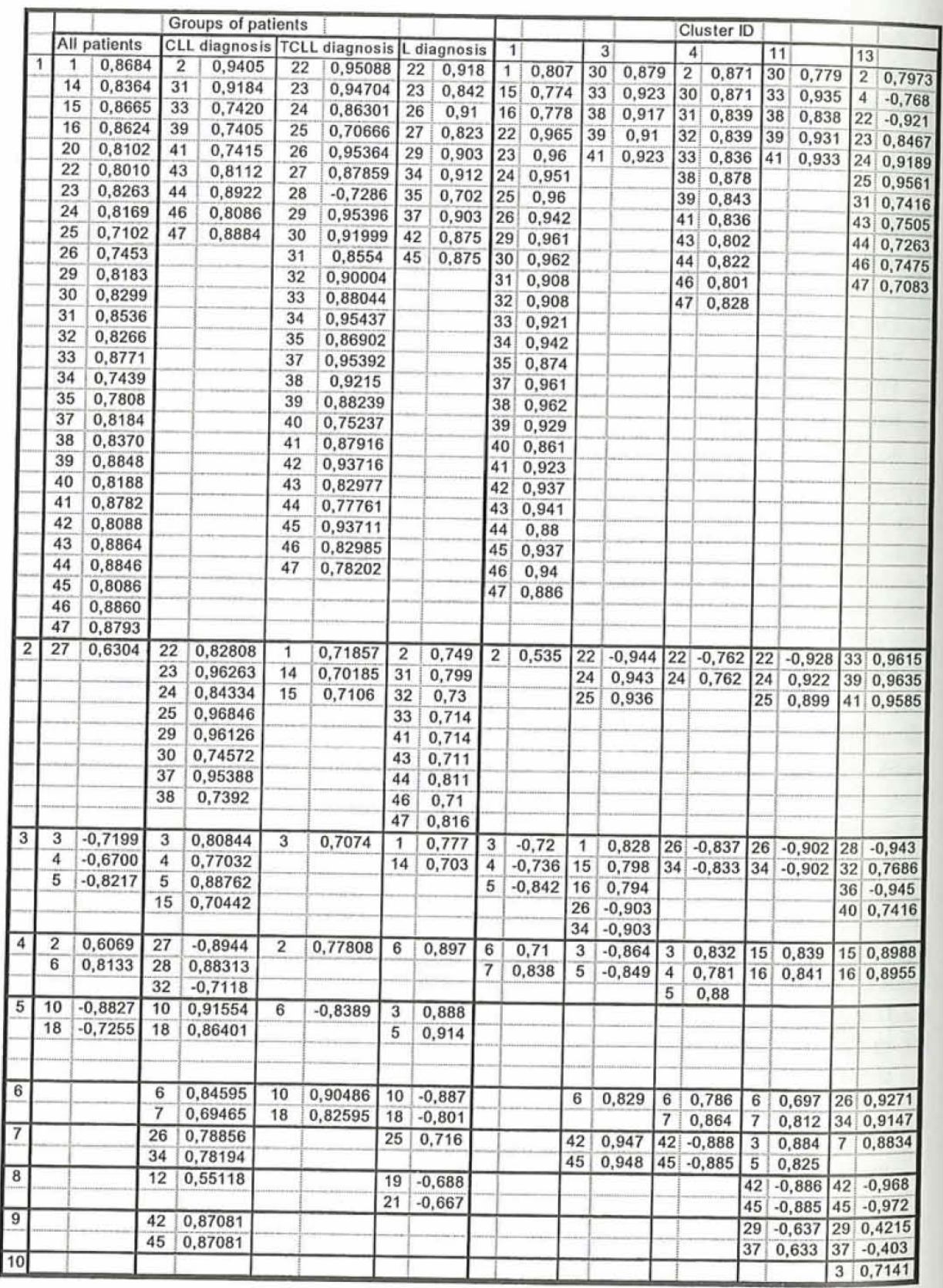

Table 1. The results of factor analysis of the features for different groups of the nuclei. First column contains factor numbers, in the rest of columns first number is the feature number while the second number is the value of the respective factor loading. 


\section{Conclusion}

The method of factor analysis, which allows reducing and structuring the initial data, proved to be efficient conformably to the problem of morphological analysis of lymphocyte nucleus. It confirmed that the proposed feature space, reflecting morphological characteristics of lymphocyte nucleus used in diagnostics, has a sufficiently simple factor structure. The main goals of factor analysis were achieved, since we succeeded in reducing the feature set composed of 47 elements at least to 8 informative factors and in making a classification of the features proposed. The important result is that the extracted factors allow to distinguish some groups of patients. This implies that received factors have contansive medical meaning. The results presented above are the prerequisites for involving factor analysis in the automated system for morphological analysis of the cytological specimens in order to create a complex model of phenomenon investigated.

In future we intend to carry out the factor analysis on the other samples of patients in supplemented feature space for the purpose of conformation of existence of extracted factors, and to exploit new methods of factor analysis as well.

\section{References}

1. Churakova, J.V., Gurevich, I.B., Hilkov, A.V., Jernova, I.A., Kharazishvili, D.V., Nefyodov, A.V., Sheval, E.V.: Selection of Diagnostically Valuable Features for Morphological Analysis of Blood Cells. Pattern Recognition and Image Analysis: Advances in Mathematical Theory and Applications. 2 (2003) $382-383$

2. Elkina, V.N., Zagoruiko, N.G.: Some Classification Algorithms Developed at Novosibirsk. In: Simon, J.C. (ed.): Intelligence Artificielle, Reconnaissance des Formes. R.A.I.R.O. Informatique/Computer Science. 1 (1978) 37-46

3. Gurevich, I.B., Khilkov, A.V., Murashov, D.M., et al.: Black Square Version 1.0: Programm Development System for Automation of Scientific Research and Education. Pattern Recognition and Image Analysis: Advances in Mathematical Theory and Applications. 4 (1999) 609-634

4. Gurevich, I.B., Harazishvili, D.V., Jernova, I.A., Nefyodov, A.V., Vorobjev, I.A.: Information Technology for the Morphological Analysis of the Lymphoid Cell Nuclei. Proceedings of the 13th Scandinavian Conference on Image Analysis (SCIA 2003), Sweden, June 29 July 2, Springer, (2003) 541-548

5. Zhuravlev, Yu.I., Gurevitch, I.B.: Pattern Recognition and Image Recognition. Pattern Recognition and Image Analysis: Advances in Mathematical Theory and Applications in the USSR. 2 (1991) 149-181 